\title{
Caracterización de líquidos de pirólisis para su posterior tratamiento mediante separación o hidrotratamiento
}

\author{
María Atienza-Martínez ${ }^{1}$, Isabel Fonts ${ }^{1,2}$, Jesús Salafranca ${ }^{3}$, Óscar de la Iglesia ${ }^{2,4}$, José \\ Luis Sánchez ${ }^{1}$ \\ ${ }^{1}$ Grupo de Procesos Termoquímicos (GPT) \\ Instituto de Investigación en Ingeniería de Aragón (I3A) \\ Universidad de Zaragoza, Mariano Esquillor s/n, 50018, Zaragoza, Spain. \\ Tel. +34-976762707, e-mail: atienza@unizar.es \\ ${ }^{3}$ GUIA-University Analytical Research Group \\ ${ }^{2}$ Centro Universitario de la Defensa - AGM \\ ${ }^{4}$ INA- Instituto de Nanociencia de Aragón
}

\section{Abstract}

En este trabajo se plantea la puesta en marcha de métodos analíticos para la caracterización química del líquido de pirólisis de cara a su utilización en el estudio de los procesos de separación y de hidrotratamiento del mismo.

\section{Introducción y objetivos}

La biomasa es una fuente renovable de carbono e hidrógeno que tiene el potencial de poder ser transformada mediante determinados procesos en combustibles que pueden sustituir parte de los actuales combustibles de origen fósil [1]. La pirólisis rápida seguida de posteriores tratamientos de mejora del líquido basados en hidrogenaciones es una de las tecnologías más prometedoras para dicho objetivo [2]. Sin embargo, se necesita todavía de un mayor desarrollo del proceso de hidrotratamiento del líquido que permita conocer con claridad sobre qué compuestos y qué grupos funcionales se debe actuar en los procesos catalíticos para que éstos sean diseñados a tal efecto.

Además de la posible utilización del líquido de pirólisis como combustible, éste contiene compuestos como azúcares, hidroxiacetaldehído, ácido acético, fenol o compuestos aromáticos, entre otros muchos, que de ser separados de una manera conveniente pueden comercializarse, ya que son productos químicos de valor añadido o pueden abrir nuevas perspectivas en el esquema de una biorrefinería [3]. Sin embargo, en un futuro próximo se debería profundizar en el estudio de los procesos de separación de productos químicos de valor añadido de la matriz del líquido de pirolisis.

Para poder avanzar tanto en los procesos de hidrotratamiento del líquido de pirólisis como en los de separación y purificación de los productos químicos de valor añadido, es de suma importancia poder disponer de métodos analíticos, con especial énfasis en los instrumentales que permitan su caracterización en profundidad de modo que se pueda conocer perfectamente cuál es el efecto de dichos tratamientos.

El líquido obtenido en la pirólisis de biomasa lignocelulósica es una combinación compleja de agua y de compuestos orgánicos provenientes de la devolatilización individual de los componentes principales de la biomasa (celulosa, hemicelulosa, lignina y extractivos), así como de reacciones secundarias entre éstos. Las diferencias en volatilidad, polaridad, peso molecular, grupos funcionales o estabilidad térmica de estos compuestos hacen imposible su caracterización mediante una única técnica instrumental. La fracción volátil analizable mediante cromatografía de gases (alrededor del 30\%) es la más conocida. Sin embargo, la identificación y cuantificación de compuestos individuales es muy complicada en el caso de los oligómeros de alto peso molecular, que debería realizarse mediante HPLC con detectores de espectrometría de masas, con el problema de que no existen espectrotecas comerciales. Por ello, en lugar de cuantificar individualmente compuestos, se puede diseñar una estrategia de caracterización que permita seguir la química básica del líquido de pirólisis y que arroje información acerca de los resultados obtenidos en post-tratamientos del líquido ya sean de hidrotratamiento o de separación de productos de valor añadido.

De acuerdo a la bibliografía [4-8], se han utilizado métodos de caracterización basados en técnicas térmicas, cromatográficas, espectrofotométricas, de espectroscopía de masas, de infrarrojo y de resonancia magnética nuclear, aunque ninguno de ellos permite una caracterización total e individual 
de los compuestos presentes. En ocasiones, los métodos combinan estas técnicas instrumentales con el pretratamiento de la muestra mediante diferentes procedimientos de extracción, destacando especialmente la utilización de extracciones líquidolíquido. Esta técnica no puede asegurar en muchas ocasiones una separación completa ya que los compuestos se distribuyen entre los disolventes utilizados y por ello, se debe seleccionar su aplicación cuidadosamente. También se han utilizado métodos basados en valoraciones para la determinación de los grupos funcionales existentes en los compuestos que forman los líquidos [8].

El objetivo de este trabajo es profundizar en la caracterización de los líquidos de pirólisis como fuente de productos químicos de valor añadido y de biocombustibles mediante la utilización de diversas técnicas analíticas.

\section{Materiales y métodos}

\section{Líquido de pirólisis}

Se ha caracterizado un líquido de pirolisis suministrado por la compañía BTG.

\section{Caracterización del líquido de pirólisis}

La caracterización del líquido de pirolisis se ha realizado mediante las siguientes técnicas: análisis elemental, poder calorífico superior (PCS), contenido en agua, cromatografía de gases acoplada a espectrometría de masas (GC-MS), fraccionamiento con disolventes (siguiendo la metodología propuesta por Oasmaa y Kuoppala [9]), contenido en extractivos (mediante extracción con hexano) y espectroscopía infrarroja por transformada de Fourier (FTIR).

\section{Resultados y discusión}

A continuación, se muestran los resultados obtenidos en la caracterización tanto del líquido de pirolisis como de las diferentes fracciones en las que se ha separado.

\section{Caracterización del líquido de pirólisis}

El líquido de pirolisis estudiado no presenta separación de fases, tiene un contenido en agua del $27,3 \%$ en masa y un PCS de $17,5 \mathrm{MJ} \cdot \mathrm{kg}^{-1}$. Se trata de un líquido con un alto contenido en carbono y oxígeno $(43,4 \%$ en masa y $49,1 \%$ en masa, respectivamente) y un bajo contenido en nitrógeno y azufre (inferior al 0,2\%).
El análisis cualitativo mediante GC-MS revela que, en términos de porcentaje de área cromatográfica, el compuesto orgánico mayoritario es el levoglucosano, habiendo también una cierta cantidad de derivados del fenol.

Los extractivos son compuestos de carácter apolar, contenidos en mayor abundancia en la corteza y las hojas de la biomasa lignocelulósica. El contenido en extractivos del líquido de pirolisis es de 1,8\%. Estos extractivos tienen un $74,2 \%$ de carbono, $8,1 \%$ de hidrógeno y $17,7 \%$ de oxígeno.

\section{Caracterización de las fracciones presentes en el líquido de pirólisis}

El $16,9 \%$ del líquido de pirolisis es insoluble en agua $y$ en diclorometano (HMW), correspondiéndose con la lignina de alto peso molecular. El 13,9\% del líquido no se disuelve en agua pero si en diclorometano (LMW), y se correspondería con la lignina de bajo peso molecular. El 27,2\% del líquido es soluble en agua e insoluble en éter (Sugars, fundamentalmente azúcares y oligómeros derivados de los azúcares), mientras que el $14,8 \%$ es soluble tanto en agua como en éter (ES) y contiene aldehídos, cetonas, furanos y monofenoles. El análisis elemental de estas fracciones se muestra en la Tabla1.

La caracterización realizada junto con información bibliográfica acerca de las posibles estructuras de los compuestos oligoméricos (lignina pirolítica [10] y humina pirolítica [11]) que contienen el líquido puede permitir determinar propiedades termodinñamicas de interés para la simulación de tratamientos posteriores de upgrading.

En la Figura 1 se puede observar los grupos funcionales más característicos de las fracciones separadas del líquido de pirólisis, analizados mediante FTIR. La fracción "Sugars", que contiene principalmente los productos derivados de la pirólisis de celulosa y hemicelulosa, muestra una mayor absorbancia alrededor de la banda característica de los grupos $\mathrm{OH}$, los cuales son muy abundantes en los monómeros de azúcares constituyentes de la celulosa y la hemicelulosa. Además, se observa una importante absorción en la fracción de los azúcares alrededor de la banda de $1049 \mathrm{~cm}^{-1}$, debido a que esa banda se corresponde con el grupo funcional C-O que es característico de los carbohidratos. Por otro lado, el ratio obtenido entre las bandas a 1049 y $1595 \mathrm{~cm}^{-1}$ puede utilizarse 
para comparar la proporción de carbohidratos y aromáticos en las fracciones analizadas. Comparando estos ratios, se puede ver que en las fracciones derivadas de la pirólisis de lignina (HMW y LMW) la proporción de aromáticos es mayor que la de carbohidratos, mientras que en la fracción de azúcares ocurre lo contrario.

\section{Conclusiones}

Se ha conseguido poner en marcha métodos analíticos que permitirán, por un lado, profundizar en el efecto de tratamientos de upgrading en la naturaleza química del mismo $\mathrm{y}$, por otro lado, determinar propiedades termodinámicas del líquido en su conjunto y de sus fracciones, que serán útiles para simular el efecto de dichos tratamientos.

\section{REFERENCIAS}

[1]. HUBER, G.W., IBORRA, S., and CORMA, A. Synthesis of transportation fuels from biomass: Chemistry, catalysts, and engineering. Chemical Reviews. 2006, 106(9), 4044-4098. Available from: doi: $10.1021 / \mathrm{cr} 068360 \mathrm{~d}$

[2]. ZACHER, A.H., OLARTE, M.V., SANTOSA, D.M., ELLIOTT, D.C., and JONES S.B. A review and perspective of recent bio-oil hydrotreating research. Green Chemistry. 2014, 16(2), 491-515. Available from: doi: $10.1039 / \mathrm{c} 3 \mathrm{gc} 41382 \mathrm{a}$

[3]. LI, X., LUQUE-MORENO, L.C., OUDENHOVEN, S.R.G., REHMANN, L., KERSTEN, S.R.A., and SCHUUR, B. Aromatics extraction from pyrolytic sugars using ionic liquid to enhance sugar fermentability. Bioresource Technology. 2016, 216(12), 12-18. Available from: doi: 10.1016/j.biortech.2016.05.035

[4]. MOHAN, D., PITTMAN, C.U., and STEELE, P.H.. Pyrolysis of wood/biomass for bio-oil: A critical review. Energy \& Fuels. 2006, 20(3), 848-889. Available from: doi: 10.1021/ef0502397

[5]. STAŠ, M., KUBIČKA, D., CHUDOBA, J., and POSPÍŠIL, M. Overview of Analytical Methods Used for Chemical Characterization of Pyrolysis Bio-oil. Energy \& Fuels. 2014, 28(1), 385-402. Available from: doi: $10.1021 / \mathrm{ef} 402047 \mathrm{y}$
[6]. LU, Q., ZHANG, Z.B., ZHANG, C.J., SU, S.H., LI, W.Y., and DONG, C.Q.. Overview of Chemical Characterization of Biomass Fast Pyrolysis Oils. Applied Mechanics and Materials. 2012, 130-134, 422-425. Available from: doi: 10.4028/www.scientific.net/AMM.130-134.422

[7]. STANKOVIKJ F, MCDONALD AG, HELMS GL, GARCIA-PEREZ M. Quantification of Bio-Oil Functional Groups and Evidences of the Presence of Pyrolytic Humins. Energy \& Fuels. 2016, 30(8), 6505-65024. Available from: doi: 10.1021/acs.energyfuels.6b01242

[8]. FERRELL, J.R., OLARTE, M.V., CHRISTENSEN, E.D., PADMAPERUMA, A.B., CONNATSER, R.M., STANKOVIKJ, F., MEIER, D., and PAASIKALLIO, V. Standardization of chemical analytical techniques for pyrolysis bio-oil: history, challenges, and current status of methods. Biofuels, Bioproducts and Biorefining. 2016, 10(5), 496-507. Available from: doi: 10.1002/bbb.1661

[9]. OASMAA, A., and KUOPPALA, E. Solvent Fractionation Method with Brix for Rapid Characterization of Wood Fast Pyrolysis Liquids. Energy \& Fuels. 2008, 22(6), 4245-4248. Available from: doi: $10.1021 / \mathrm{ef} 800407 \mathrm{~d}$

[10].FRATINI, E., BONINI, M., OASMAA, A., SOLANTAUSTA, Y., TEIXERA, J. and BAGLIONI, P. SANS Analysis of the Microstructural Evolution during the Aging of Pyrolysis Oils from Biomass. Langmuir. 2006, 22(1), 306-312. Available from: doi: $10.1021 / \mathrm{la} 051990 \mathrm{a}$

[11].STANKOVIKJ, F., MCDONALD, A.G., HELMS, L.G., OLARTE, M.V., and GARCIA-PEREZ, M. Characterization of the Water-Soluble Fraction of Woody Biomass Pyrolysis Oils. Energy \& Fuels. 2017, 21(2), 1650-1664. Available from: doi: 10.1021/acs.energyfuels.6b02950 
Tabla 1. Caracterización de las fracciones del líquido de pirólisis

\begin{tabular}{|c|c|c|c|}
\hline & HMW & LMW & Sugars \\
\hline $\begin{array}{c}\text { Carbono ( } \% \text { en } \\
\text { masa) }\end{array}$ & 64,3 & 66,6 & 44,3 \\
\hline $\begin{array}{l}\text { Hidrógeno ( } \% \\
\text { en masa) }\end{array}$ & 5,9 & 6,6 & 6,5 \\
\hline $\begin{array}{l}\text { Nitrógeno (\% en } \\
\text { masa) }\end{array}$ & 0,2 & $<0,1$ & 0,1 \\
\hline $\begin{array}{c}\text { Oxígeno ( } \% \text { en } \\
\text { masa) }\end{array}$ & 29,6 & 26,8 & 49,0 \\
\hline PCS $\left(\mathrm{MJ} \cdot \mathrm{kg}^{-1}\right)$ & $25,0^{*}$ & 26,6 & 17,3 \\
\hline
\end{tabular}

* Calculado con la fórmula de Dulong.

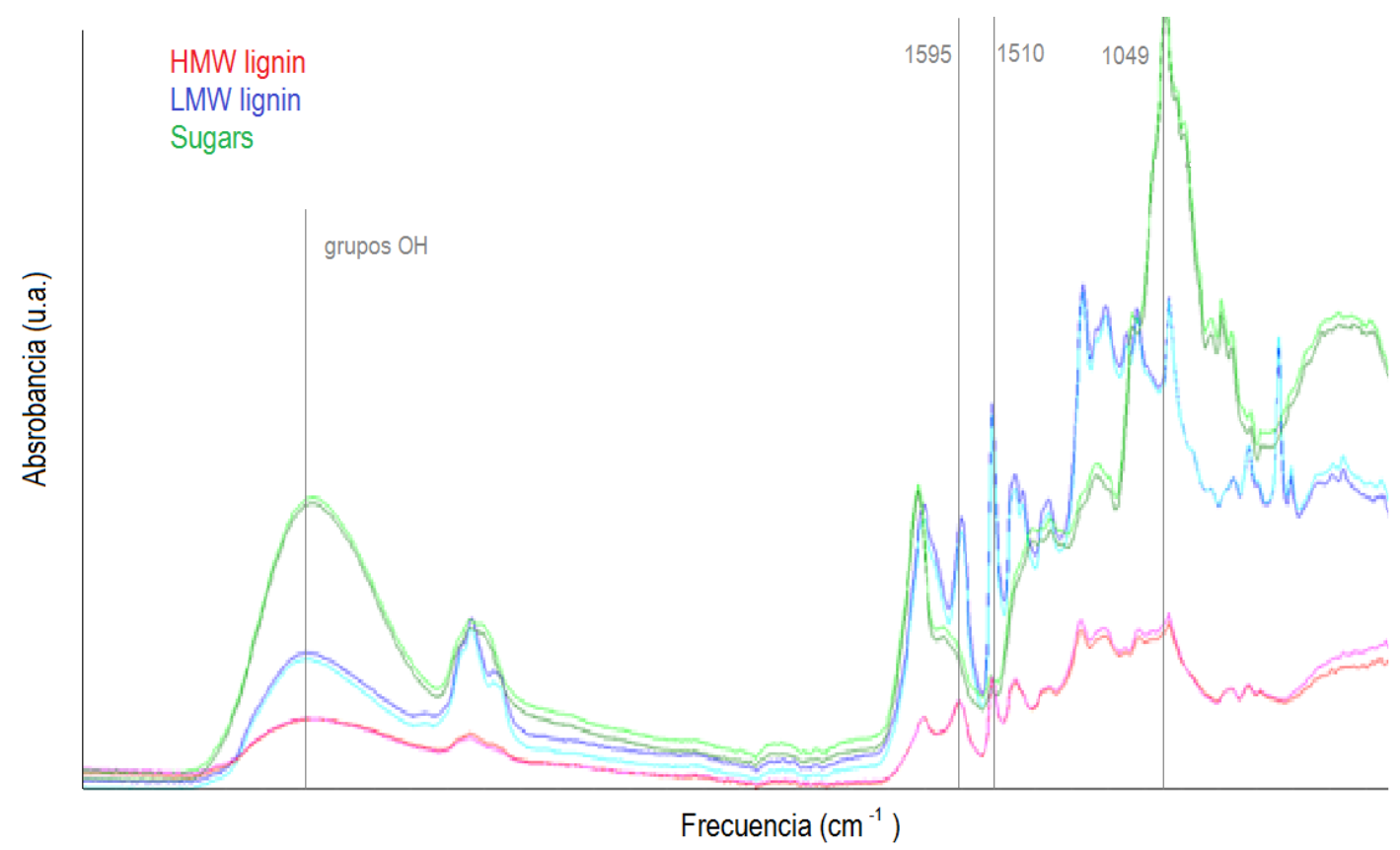

